\title{
Editorial
}

\section{Multimedia Transmission over Emerging Wireless Technologies}

\author{
Stavros Kotsopoulos ${ }^{1}$ and Tasos Dagiuklas ${ }^{2}$ \\ ${ }^{1}$ Department of Electrical and Computer Engineering, University of Patras, 26504 Patras, Greece \\ ${ }^{2}$ Department of Telecommunication Systems and Networks, TEI of Mesolonghi, 30300 Nafpaktos, Greece
}

Received 8 January 2008; Accepted 9 January 2008

Copyright (c) 2007 S. Kotsopoulos and T. Dagiuklas. This is an open access article distributed under the Creative Commons Attribution License, which permits unrestricted use, distribution, and reproduction in any medium, provided the original work is properly cited.

The efficient delivery of multimedia applications and services over emerging diverse and heterogeneous wireless networks (e.g., 2.5G/3G/4G, Wi-Max, WLANs, PANs, ad hoc, etc.) is a challenging research objective. The research effort for the $3 \mathrm{G} / 4 \mathrm{G}$ vision of interworking among heterogeneous technologies to achieve multimedia session continuity, retain multimedia QoS characteristics, and so forth amplifies the need to evaluate the conditions and restrictions under which delivery of such services can be accomplished. Furthermore, there are several research issues such as encoding techniques, cross-layer optimization, error control, security require considerable researcheffort so that appropriate solutions are designed and developed. The objective of this Special Issue is to present state-of-the-art research and developing activities contributing to all facets of multimedia across heterogeneous emerging wireless technologies (e.g., 4G, ad hoc, Wi-Max, etc.). This Special Issue aims to disseminate state-of-the-art research, development, and novel solutions of multimedia transmission over wireless/mobile-networked multimedia ad hoc systems and technologies.

The first paper entitled "WLAN technologies for audio delivery" by N. Tatlas et al. presents audio delivery over WLAN and investigates the synchronized and robust realtime streaming of multiple audio channels to multipoint receivers, for example, wireless active speakers. A novel synchronization scheme is also introduced, allowing optimized playback for multiple receivers. The perceptual audio performance is assessed for both stereo and 5-channel applications based on either PCM or compressed audio signals.

The second paper entitled "Efficient TTI for 3G multimedia applications" by C. Chaikalis addresses time transmission interval (TTI), which is a very important task for implementing optimum UMTS turbo coding in flat Rayleigh fading environment. Different multimedia scenarios are investigated by C. Chaikalis, using maximum UMTS frame length. It is shown that different operating environments require appropriate TTI in terms of BER performance for a variety of data rates.

The impact of data transfer service, multimedia streaming service, web service, and traffic load on speech quality in an environment of WLANs (IEEE 802.11) has been examined at the third paper entitled "Impact of background traffic on speech quality in VoWLAN" by P. Pocta et al. In their paper, a new method for improving the detection of the critical conditions in wireless networks from the speech quality point of view is presented.

The forth paper entitled "Fuzzy logic control of adaptive ARQ for video distribution over a Bluetooth wireless link" by R. Razavi et al. considers adaptive ARQ, suitable for video transmission over Bluetooth links. The authors present a fuzzy logic control of ARQ, based on send buffer fullness and the head-of-line packet's deadline. The scheme considers both the delay constraints of the video stream and at the same time avoids send buffer overflow. Tests explore a variety of Bluetooth send buffer sizes and channel conditions.

The fifth paper entitled "Utilizing cross-layer information to improve performance in JPEG2000 decoding" by $\mathrm{H}$. Persson et al. focuses on wireless multimedia communication and investigates the scenario of how the cross-layer information can be used to improve the performance in image quality at the application layer using the IPEG2000 standard.

The sixth paper entitled "Joint optimization in UMTSbased video transmission" by A. Zsiros et al. presents a new software platform to enable demonstration and capacity testing within the framework of IST-PHOENIX project. The developed platform simulates a joint optimized UMTS video transmission. 
The seventh paper entitled "Energy-constrained quality optimization for secure image transmission in wireless sensor networks" by W. Wang et al. proposes a new cross layer approach to optimize image transmission quality for secure digital image delivery in wireless sensor networks (WSNs) with strict energy budget constraint. First, a selective encryption approach favorable for UEP communication schemes with cipherplain-text diversity as well as position-value ( $\mathrm{P}$ $\mathrm{V}$ ) diversity is proposed. Network resources such as desirable BER, ARQ retry limit, and transmission rates are jointly optimized across PHY, MAC, and APP layers regarding distortion reduction distribution bounds and inter-segment correlation for image data transmission.

Metamodeling comparison issues are involved in the eight paper entitled "A comparison performance analysis of QoS WLANs: approaches with enhanced features" by I. Papapanagiotou et al. The contribution of their paper is to compare and enhance known methods for performance analysis of the IEEE 802.11e MAC layer such as Markov chains, queuing theory, and probabilistic analysis. The proposed analyses carry by themselves scientific interest because they are extended enhancements with the latest enhanced distribution coordination (EDCA) parameters.

The ninth paper entitled "Video broadcasting using queue proportional scheduling" by D. Toumbakaris and S. Kotsopoulos examines the use of queue proportional scheduling for video broadcasting. First, the behavior of QPS is examined as the scheduling frequency is reduced and a method is proposed that uses statistics on the arrival rates to improve its performance. The reduction of the scheduling frequency simplifies the scheduler and decreases the required operations. Using a Markov chain to model packet delay distribution, it is discussed how the video encoding rate can be chosen in order to reduce the expected distortion of streams transmitted through broadcast channels.

The tenth paper entitled "Distortion optimized packet scheduling and prioritization of multiple video streams over 802.11e networks" by I. Politis et al. presents a generic framework solution for minimizing video distribution of multiple video streams transmitted over 802.11 e wireless networks. Their work includes intelligent packet scheduling and channel access differentiation mechanisms. Two intelligent scheduling algorithms have been proposed that will select which packet or combinations of packets over all video streams will be dropped based on the available transmission rate of the communication network, which in this case is 802.11e WLAN.

Stavros Kotsopoulos Tasos Dagiuklas 

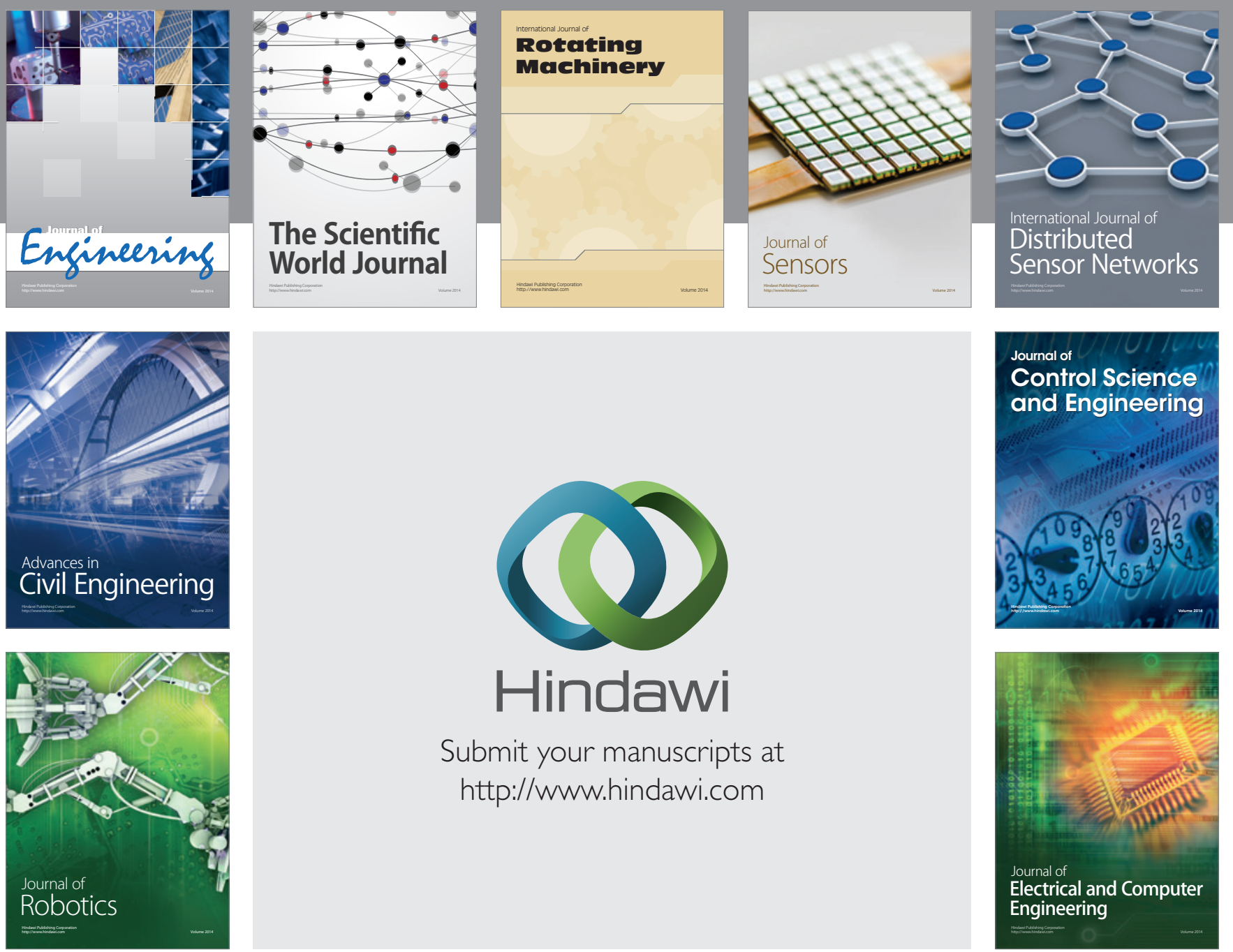

Submit your manuscripts at

http://www.hindawi.com
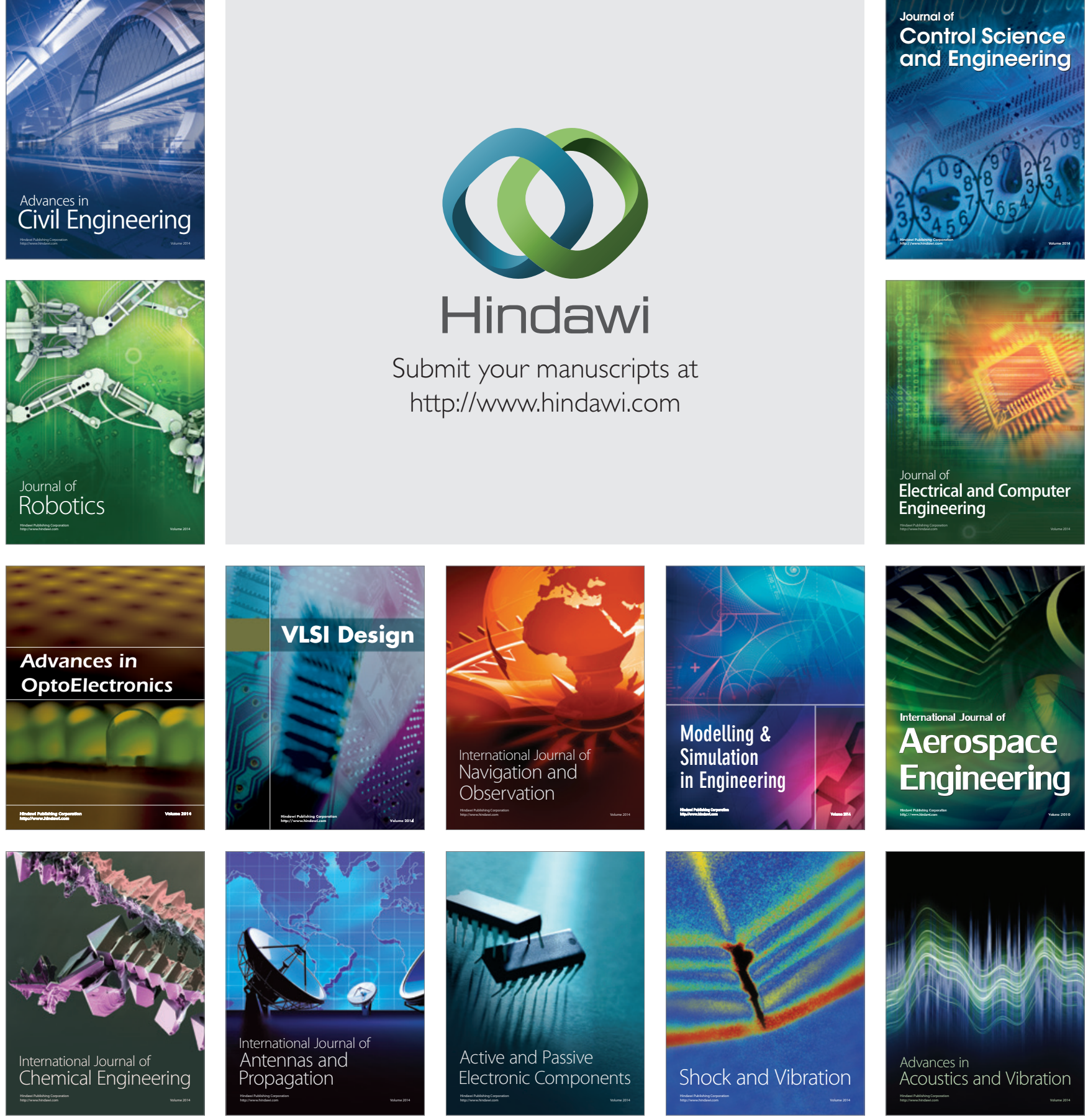